\title{
Decompression Surgery With Antibiotic- impregnated Calcium Sulfate Can Achieve More Satisfactory Results Than Decompression Alone in Pediatric Hematogenous Osteomyelitis
}

\section{Cheng-he Qin ( $D$ orthoqin@163.com )}

the Second Clinical Medical School of Southern Medical University

Rui Tao

Southern Medical University Nanfang Hospital

\section{Yu-han Chee}

National University of Singapore

Ji-wei Luo

Southern Medical University Nanfang Hospital

\section{Lei $\mathrm{Xu}$}

the Second Clinical Medical School of Southern Medical University

Jia Fang

the Second Clinical Medical School of Southern Medical University

Yan-jun Hu

Southern Medical University Nanfang Hospital

Chun-hao Zhou

Southern Medical University Nanfang Hospital

\section{Research Article}

Keywords: hemotogenous osteomyelitis, pediatric,calcium sulfate, hospital stay, wound healing duration, infection recurrence, pathological fracture, leakage of the incision

Posted Date: March 16th, 2021

DOl: https://doi.org/10.21203/rs.3.rs-289026/v1

License: (1) This work is licensed under a Creative Commons Attribution 4.0 International License.

Read Full License 


\section{Abstract}

\section{Background}

The curative effect of antibiotic-impregnated Calcium Sulfate in pediatric ostemyelitis is unknown. The purpose of this study was to compare the outcomes of two treatment methods, decompression combined with antibiotic-impregnated calcium sulfate and decompression alone, for the treatment of pediatric Hemotogenous Osteomyelitis.

\section{Methods}

Between 2013 to 2016, forty-one patients with Hematogenous Osteomyelitis met the criteria were included for assessment. Twenty-one patients were included in the calcium sulfate group (the CS group) in which vancomycin and/or gentamicin impregnated calcium sulfate was used as an adjuvant after bone decompression while 20 patients as the control group were undergone bone decompression alone. The infection recurrence rate, hospital stay, wound healing duration, serum inflammatory index, pathological fracture, leakage of the incision, follow-up time were compared between the two groups.

\section{Results}

Infection recurrence was 0\% (0/21) in CS group and 15\%(3/20) in control group within 12 months $(\boldsymbol{P}=0.107)$. Infection recurrence was $0 \%(21 / 21)$ in CS group and $20 \%(16 / 20)$ of patients at a minimum of 24(range 24 to 67 ) months follow-up $(\boldsymbol{P}=0.048)$. Mean hospital stay were 8.19 (range 3 to 21 ) days in CS group and 15.95(range 5 to 47 ) days in control group $(\boldsymbol{P}=0.02)$. Mean wound healing duration were 16.1(range 10 to 29 ) days in CS group and 15.5(range 10 to 25 ) days in control group $(\boldsymbol{P}=0.65)$. Serum inflammatory index for WBC(White Blood Cells), ESR( Sedimentation Rate), CRP(C-Reactive Protein) was respectively 8.76(6.03-12.07)*10^9/L, 44.14(26-70)mm/L, 12.35(1.03-35.04) mg/Lin CS group and $8.90(5.68-13.56) * 10^{\wedge} 9 / \mathrm{L}, 39.25(19-57) \mathrm{mm} / \mathrm{L}, 15.65(1.02-45.69) \mathrm{mg} / \mathrm{L}$ in control group $(\boldsymbol{P}=0.82$, $\boldsymbol{P}=0.31, \boldsymbol{P}=0.51)$. Pathological fracture was $0 \%(0 / 21)$ in CS group and $10 \%(2 / 20)$ in control $\operatorname{group}(\boldsymbol{P}=0.23)$. Leakage of the incision was $4.76 \%(0 / 21)$ in CS group and $10 \%(2 / 20)$ in control $\operatorname{group}(\boldsymbol{P}=0.61)$.

\section{Conclusions}

Antibiotic-impregnated Calcium Sulfate can reduce infection recurrence and shorten the mean hospital stay in pediatric Hemotogenous Osteomyelitis. The curative effect of antibiotic-impregnated Calcium Sulfate in pediatric ostemyelitis is satisfactory.

\section{Trial registration}

Retrospectively registered.

\section{Background}


Calcium Sulfate (CS) is a representative of bioabsorbable bone substitute, which was widely used in bone infection when loaded antibiotic.[1-6]. Existing clinical articles have focused on the efficacy of antibioticimpregnated CS in post-traumatic osteomyelitis, infection after internal fixations and infected nonunion. [7-10] However, the efficacy of antibiotic-impregnated CS in pediatric Hematogenous Osteomyelitis $(\mathrm{HO})$ is unclear. $\mathrm{HO}$ is different from non-HO both in pathogenesis and bacteriology.[11] The characteristics of $\mathrm{HO}$ include spread by hematogenous route[12], dominance of staphylococcus aureus.[13] A majority of pediatric $\mathrm{HO}$ can be cured by appropriate antibiotics administered sequentially via parenteral and oral routed. $[12,14,15]$ Surgical is necessary when invalid intravenous antibiotic. However, pediatrics is different from adult due to the epiphyseal plate, the main purpose for surgery is decompression[13] in pediatrics compare with radical debridement[16] in adult. Local antibiotics delivery, with vancomycin or tobramycin impregnated CS, has been widely used in osteomyelitis due to its decent efficacy in adult, such as reducing infection recurrence and shortening treatment cycle.[7, 8] A recent research article reported that tobramycin-impregnated CS pellets can reduce the risk of associated comorbidities, hospital stays, and healthcare costs in the treatment of HO in children and adolescents.[10] However, few comparative studies had been carried out to confirm those results. In this study, we want to find the differences of infection recurrence rate, hospital stay, postopreative serum inflammatory markers, wound healing rate, pathological fracture and leakage of the incision when added this local antibiotic delivery.

\section{Methods}

\section{Study Design and Setting}

A retrospective study was performed analyzing pediatric $\mathrm{HO}$ patients treated in our hospital from January 2013 to December 2016. The main inclusion criteria were as follows: 1) pediatric patients aged below 18 years old. 2) patients with $\mathrm{HO}$ underwent surgical bone decompression. 3) patients persisted to the follow-up and had been followed for at least 24 months. The main exclusion criteria included: 1) patients suffered from direct spreading osteomyelitis, such as post-traumatic osteomyelitis, or infection after internal fixation. 2) patients received a soft tissue surgery, 3) follow-up losted patients. The collectiong clinical data include name, sex, age, diagnosis, serum inflammatory indexs (White blood cell count, Erythrocyte Sedimentation Rate, C-reactive protein), bacteriology, imaging data, site of osteomyelitis, operation process, discharge date, surgery date, telephone number and duration of follow-up .

\section{Description of Study Population}

Forty-one patients met the criterion and were included in our study. (Figure 1) Twenty-one patients (13 boys, 8 girls), a mean age of 10.14 years old (range, 3-18 years old), received decompression surgery with antibiotic-impregnated CS were allocated to CS group. Twenty children (17 boys, 3 girls), a mean age of 11.85 years old (range, 2-18 years old), received decompression surgery alone were allocated to control group. (Table 1) All patients had no draining sinuses. All patients had no comorbidities such as sickle cell anemia, deep vein thrombosis, septic pulmonary embolism, pneumonia,empyema and endocarditis. 
Table 1. Demographics of Hemotogenous Osteomyelitis patients.

\begin{tabular}{|c|c|c|c|}
\hline & CS Group † & Control Group & $p$ value \\
\hline \multicolumn{4}{|l|}{ Sex } \\
\hline boy & 13 & 17 & \multirow[t]{2}{*}{0.159} \\
\hline girl & 8 & 3 & \\
\hline Age (years old) & $10.14(3-18)$ & $11.85(2-18)$ & 0.226 \\
\hline Course of disease (days) & $53(8-127)$ & $59(10-110)$ & 0.92 \\
\hline Patients with fever & 18 & 12 & 0.085 \\
\hline Days of fever & $7(3-14)$ & $8(4-12)$ & 0.448 \\
\hline \multicolumn{4}{|l|}{ Bacteriology } \\
\hline MRSA $\ddagger$ & 5 & 4 & \multirow[t]{2}{*}{1.00} \\
\hline Non MRSA & 16 & 16 & \\
\hline Staphylococcus aureus & 10 & 10 & \\
\hline Enterobacter cloacae & 1 & 0 & \\
\hline Negative & 5 & 6 & \\
\hline \multicolumn{4}{|l|}{ Previous operation history $£$} \\
\hline Yes & 8 & 4 & \multirow[t]{2}{*}{0.306} \\
\hline NO & 13 & 16 & \\
\hline White blood cell count $\left({ }^{*} 10^{\wedge} 9 / \mathrm{L}\right)$ & $9.47(5.99-21.78)$ & 10.05(5.46-25.46) & 0.682 \\
\hline Erythrocyte Sedimentation Rate $(\mathrm{mm} / \mathrm{L})$ & $37.86(3-102)$ & $34.60(5-85)$ & 0.695 \\
\hline $\begin{array}{l}\text { C-Reactive } \\
\text { protein (mg/L) }\end{array}$ & $28.21(0-87.18)$ & $26.70(0.2-107.8)$ & 0.909 \\
\hline
\end{tabular}

† CS, Calcium Sulfate

‡ MRSA, Methicillin-Resistant Staph. Aureus

$£$ Previous operation history: a biopsy surgery

\section{Description of surgery}


The deep soft-tissue fluid collections or abscesses and subperiosteal were removed. Decompression was performed by creating an appropriately placed cortical window in long bone osteomyelitis, followed by curettage of intraosseous abscess. Eggshell-like decompresion technology[17] was used in calcaneus osteomyelitis, followed by curettage of abscess.

Then difference between CS group and control group: The former one involves antibiotic-impregnated CS application to obliterate the dead space and release local antibiotic. $1 \mathrm{~g}$ vancomycin (or $160 \mathrm{mg}$ gentamicin or both) and $10 \mathrm{cc}$ calcium sulfate were mixed thoroughly using the solvent provided by the manufacturer until a smooth paste is formed (approximately $30 \mathrm{~s}$ ). Calcium sulfate (beads or blockshaped or both) is then placed or filled into the defect as well as supero-inferior medullary cavity. (Figure 2) The paste is allowed to cure undisturbed for at least $15 \mathrm{~min}$ after mixing. The volume of calcium sulfate varied according to the size of the bone defect. No drainage tube was used. The later one without this antibiotic material, an irrigation tube was placed in the lower medullary cavity and a drainage tube was placed in the upper medullary cavity. (Figure 3) If the pus is small enough, we place a drainage tube only. In both CS group or control group, a cast or external fixation were applied if bone is unstable after decompression.

\section{Aftercare}

All patients were routinely given intravenous antibiotics post operation. Cefmetazole, $100 \mathrm{mg} / \mathrm{kg} / \mathrm{day}$, was chosen in most patients. Clindamycin, $30 \mathrm{mg} / \mathrm{kg} /$ day, was chosen in a few patients who were allergic to cefmetazole. Vancomycin, $40 \mathrm{mg} / \mathrm{kg} /$ day, was chosen when the pathogenic bacterium showed resistance to cefmetazole. Postoperative antibiotic including cefmetazole (34 cases), clindamycin (7 cases), vancomycin (9 cases), some patients received combinations of medications. In CS group, intravenous antibiotics were administered for a mean of 10.9 days followed by oral antibiotics for a mean of 15.2 days. In control group, intravenous antibiotics were administered for a mean of 12.3 days followed by oral antibiotics for a mean of 16.4 days. In CS group, cleaning the wound regularly by anerdian skin disinfectant. In control group, normal saline solution was used to irrigate by continuous flow every day. No adjuvant dilute Betadine (povidone-iodine) wash or antibiotic powder in the wound was used. The irrigation tube was removed when the fluid looks clear and the drainage tube was removed when the daily output is less than $5 \mathrm{ml}$. The patients were released when their symptoms improved and the drain removed.

\section{Outcome Measures}

The patients are regually reviewed in the outpatient department. Defined outcome parameters included infection recurrence, hospital stay, wound healing duration, serum inflammatory markers, pathological fracture, leakage of the incision and follow-up time. Infection recurrence was defined as a worsening clinical symptoms, an continuously increasing serum inflammatory markers, formation of sinus tract 
and(or) a continuing to be eroded bone tissue by X-ray. Hospital stay is calculated based on the number of days following surgery till discharge. Wound healing duration was defined as the postoperation till incision healing with no exudation and suture removed. Pathological fracture was defined fractures without apparent violence.

\section{Statistical Analysis}

Statistical analysis was performed using the SPSS (Version 13) software package (SPSS Inc, Chicago, IL). Descriptive statistics were conducted for all variables. Continuous variables were expressed as the mean standard deviation or the minimum and the maximum depending on data distribution. Student ttest was used for continuous variables. Nonparametric test was used for variance inhomogeneity. A chi square test was used for categorical variables between the two groups. Fisher exact test was used in cases in which one or more of the expected variables was less than five. A value of $p<0.05$ was considered to be statistically significant.

\section{Results}

The locations of HO were 13 in tibial (5 in CS group), 9 in femur (6 in CS group), 6 in humerus (2 in CS group), 5 in calcaneus (3 in CS group), 4 in fibula (3 in CS group), 2 in clavicle (1 in CS group), 1 in ulna in control gruop, 1 in radius in CS group. The outcomes of two groups were presented in table 2 .

Table 2. The outcomes of two groups

\begin{tabular}{|llll|}
\hline & CS group & Control group & $\begin{array}{c}\boldsymbol{p} \\
\text { value }\end{array}$ \\
\hline Recurrence within 12 months & $0 \%(0 / 21)$ & $15 \%(3 / 20)$ & 0.107 \\
\hline $\begin{array}{l}\text { Recurrence at an minimum of } 24 \\
\text { months }\end{array}$ & $0 \%(0 / 21)$ & $20 \%(4 / 20)$ & 0.048 \\
\hline Mean hospital stay (days) & $8.19(3-21)$ & $15.95(5-47)$ & 0.02 \\
\hline Mean wound healing duration (days) & $16.1(10-29)$ & $15.5(10-25)$ & 0.65 \\
\hline White blood cell count(*10^9/L) & $8.76(6.03-12.07)$ & $8.90(5.68-13.56)$ & 0.82 \\
\hline Erythrocyte Sedimentation Rate(mm/L) & $44.14(26-70)$ & $39.25(19-57)$ & 0.31 \\
\hline C-reactive protein (mg/L) & $12.35(1.03-35.04)$ & $15.65(1.02-45.69)$ & 0.51 \\
\hline Pathological fracture & $0(0 / 21)$ & $10 \%(2 / 20)$ & 0.232 \\
\hline Leakage of the incision & $4.76 \%(1 / 21)$ & $10 \%(2 / 20)$ & 0.606 \\
\hline Average Follow-up time & $34.71(24-67$ SD & $40.10(24-77$ SD & 0.18 \\
\hline
\end{tabular}


In CS group, infection recurrence was $0 \%$ (0 of 21) within 12 months. Infection recurrence was $0 \%$ (0 of 21) at an average 34.71 (SD 11.19, range 24 to 67 ) months follow up. The mean hospital stay were 8.19 (SD 4.82, range 3 to 21) days. The mean wound healing duration were 16.1 (SD 4.89, range 10 to 29) days. Serum inflammatory index for WBC, ESR, CRP was respectively $8.76(6.03-12.07) \star 10^{\wedge} 9 / \mathrm{L}, 44.14(26$ $70) \mathrm{mm} / \mathrm{L}, 12.35(1.03-35.04) \mathrm{mg} / \mathrm{L}$. Pathological fracture was $0 \%(0 / 21)$. Leakage of the incision was $4.76 \%(0 / 21)$.

In control group, infection recurrence rate was $15 \%$ (3 of 20) within 12 months (a tibia, a ulna, a humerus). Infection recurrence was was $20 \%$ (4 of 20) of patients at an average 40.10 (SD 14.06, range 24 to 77) months follow up (a additional recured femur). The mean hospital stay were 15.95 (SD 9.76, range 5 to 47) days. The mean wound healing duration were 15.5 (SD 3.99, range 10 to 25) days. Serum inflammatory index for WBC, ESR, CRP was respectively 8.90(5.68-13.56)*10^9/L, 39.25(19-57)mm/L, $15.65(1.02-45.69) \mathrm{mg} / \mathrm{L}$. Pathological fracture was $10 \%(2 / 20)$. Leakage of the incision was $10 \%(2 / 20)$.

Patients in CS group had a lower infection recurrence rate compare with those in control $(p=0.048)$. Patients in CS group had a shorter hospital stay compare with those in control $(\boldsymbol{p}=0.02)$. The wound healing duration between the two groups showed no significantly different $(\boldsymbol{p}=0.65)$. Serum inflammatory indexs in two groups for WBC, ESR, CRP showed no significantly different $(\boldsymbol{p}=0.82, \boldsymbol{p}=0.31, \boldsymbol{p}=0.51)$. Pathological fracture in two groups showed no significantly different $(\boldsymbol{p}=0.23)$. Leakage of the incision in two groups showed no significantly different $(\boldsymbol{p}=0.61)$.

\section{Discussion}

Hematogenous osteomyelitis $(\mathrm{HO})$ can be cured by intravenous antibiotic in most of teenagers and children.[18] Therefore, most researchers concentrated on early diagnosis, antibiotic therapy and interdisciplinary cooperation instead of the right timing and method of surgery.[12,18] No consensus has been reached on the timing and method of surgery so far. Copley presented a Severity of Illness Score[19] for pediatric acute $\mathrm{HO}$ in 2016. He put CRP in a prominent position and indicated that patients may require multiple surgical procedures when severe scores was 8 10.[12] However, in our experience, surgery were considered candidates when exist with abscesses or fail to respond to antibiotic therapy after 48 hours to 72 hours. Otherwise, the positive rate and accuracy rate were highest in intraoperative specimen culture than interventional radiology culture and blood culture.[18] A positive and accuracy culture is crucial for diagnosing osteomyelitis. Hence,to some extent, surgery is both diagnosis and treatment. In terms of methods of surgery, Copley put forward "decompression and drainage" in 2009.[20] The question of whether obliterate the dead space left by bone decompression while concurrently inducing high local antibiotic levels is currently a topic of scrutiny. Most prior studies have demonstrated that antibiotic-impregnated CS has high clinical efficacy in infection eradication and plays a permissive role in the formation of new bone in chronic osteomyelitis.[1, $7,8,10]$ But there was no related control trail comparing the efficacy of antibiotic-impregnated CS with nothing in pediatric $\mathrm{HO}$. We believe this retrospective control clinical trial provide stronger evidence for clinical efficacy of antibiotic-impregnated $\mathrm{CS}$ in pediatric $\mathrm{HO}$. 
Although any water-soluble antibiotic can be incorporated into the calcium sulfate,the ideal choice of antibiotic remains controversial. The choice of local antibiotic was empirical depending on local epidemiological data in patients who had no accurate bacterial data. We chose vancomycin in gram staining positive cases or in highly suspected staphylococcus aureus cases and gentamicin in gram staining negative cases. We chose vancomycin and gentamicin together in hard-to-identify bacterial cases. Compared with long-term intravenous antibiotics, local antibiotic had its unique advantages. One is effective and higher antibiotic concentrations are obtained in the local area of infected bone through the systems for a prolonged period of time. The other is to prevent the adverse events related to systemic chemotherapy and reduce the risk of systemic toxicity.[21] Zhang et al[22] measured the blood vancomycin levels in 24 osteomyelitis patients locally applied with vancomycin-impregnated calcium sulfate beads (range from $1.5 \mathrm{ml}$ to $5 \mathrm{ml}$ with a ratio of $1 \mathrm{~g}$ vancomycin: $5 \mathrm{ml}$ calcium sulfate). The results showed that the mean blood vancomycin level was still within a safe range for application. P. Wahl et al[23] found that even $6 \mathrm{~g}$ vancomycin was applied locally, the systemic concentration remained within a safe range and local concentration was still below the reported cell toxicity thresholds. In our study, patients in two groups received intravenous and oral routed antibiotic. Adverse events related to systemic chemotherapy and systemic toxicity do not happened in two groups.

Davis WT et al[24] reported multiple surgeries were underwent in Methicillin-resistant Staphylococcus aureus(MRSA) patients. In their's research, they emphasize that improving treatment for MRSA osteomyelitis should involve reevaluation of surgical techniques, such as utilizing local antibiotics. In our study, no patient recured in CS group including 5 cases of MRSA. Four patients recured in control group including 3 cases of MRSA and 1 case of negative culture.(Figure 4) Local antibiotics has exhibited excellent curative effect especially in MRSA infection. It is known to us, successful surgical eradication of osteomyelitis requires the use of antibiotics. Systemic antibiotic treatment cannot achieve sufficient release of antibiotic in the area of infected bone due to poor blood supply resulting from soft tissue scarring and bone sclerosis.[8, 25] A residual focus of infection may result in a new infection in the area of a low local bactericidal concentration of antibiotics. Moreover, long-term usage of antibiotics can promote the growth of bacterial drug resistance.[26] Prior research shows that the infections evolved to have greater invasive properties and were associated with substantial abscess formation in the era of MRSA.[24] Hence, this may lead to more surgical drainage procedures. Inserting antibiotic at the site of infection has a lot of potential merit,such as higher antibiotic concentrations in the local area of infected bone through the systems for a prolonged period of time.[8] The extremely high local concentrations released by the implant can kill the drug-resistant bacteria including the residual focus of MRSA infection. In addition, a residual focus of infection may result in a continued bone destruction and then pathological fracture. Regardless of local antibiotic, CS can promote bone healing either and increase bone strength to avoid pathologica fractures. What's more, the efficacy of antibiotic impregnated CS in $\mathrm{HO}$ is different from its efficacy in non-HO. Michael D. McKee et al[7] found that infection was eradicated in $86 \%$ (12 of 14) of patients in non-HO osteomyelitis when treated with antibiotic-impregnated CS. Our study found that infection was eradicated in $100 \%$ ( 21 of 21 ) of children diagnosed with $\mathrm{HO}$ when treated with this 
method. This result may indicate that antibiotic-impregnated CS present better eradication of infection in $\mathrm{HO}$ than non-HO.

Although difference in post operative management may explain the difference in hospital stay. But exactly the filling of the antibiotic cement result in different post operative management. It is also a potential merit of inserting antibiotic at the site of infection. Andreacchio et all[10] reported that tobramycin-impregnated CS can reduce the hospital stays in pediatric HO. In our study, we also found the use of antibiotic-impregnated CS can obviously reduce the hospital stays in pediatric $\mathrm{HO}$ compare with nothing.

CS simillary has its disadvantage, such as wound complication.[27-29] Wound complication may resulting in a delayed healing incision. A commonly reported observation associated with the use of calcium sulfate when used surgically is a fluid discharge from the wound/surgical site, occurring in $4 \%$ to $51 \%$ of cases.[7, 30,31] The reported duration of fluid discharge is variable, ranging from 2 to 24 weeks duration.[31] In our study, one patient in CS group had a aseptic exudation. This lead to a delayed stitches removing in 29 days post operation. However, in our study, the application of CS did not prolong wound healing duration compare with nothing. $(\boldsymbol{p}=0.65)$ How to prevent aseptic exudation of antibioticimpregnated CS is an urgent problem to be solved. In view of this, we present our own experience: 1. Keep the CS from getting too wet; 2 . Place the CS in the area rich in soft tissue.

Masquelet[32] proposed two steps operation technique for the treatment of chronic osteomyelitis. First step is a sharp debridement of necrotic tissue, abundant lavage, sequestrectomy, and removal of any hardware and/or foreign bodies, followed by insertion of antibiotic-impregnated Polymethylmethacrylate(PMMA). Second step is an additional surgical procedure required for removal of the beads and subsequent bone grafting. This technique can be used in case of initial infection and to control established infection before final bone reconstruction. Canavese et al.[33] reported successful treatment of 5 cases chronic pediatric osteomyelitis with debridement, antibiotic-laden PMMA and bone graft substitute. Four cases were infected after internal fixation and one case was not mentioned initiating event. Bar-On et al.[34] reported successful treatment of 4 cases chronic pediatric osteomyelitis by intramedullary reaming and antibiotic-impregnated PMMA. Two cases were infected after internal fixation and just two cases were infected hematogenous. Masquelet technique may not suitable in $\mathrm{HO}$ because of its big surgical trauma and additional additional surgical procedure. In our study,the insertion of CS did not result in a big surgical trauma and additional additional surgical procedure. However, unfortunately, we don't have any cases to compare the effiency in pediatric HO between the two methods.

\section{Limitations}

Our study was not a a prospective, randomized clinical trial. Thus a large-sample randomized controlled clinical trial should be necessary to evaluate the effect of the antibiotic-impregnated CS in the treatment for pediatric $\mathrm{HO}$. 


\section{Conclusion}

Antibiotic-impregnated CS can reduce infection recurrence and shorten the mean hospital stay in pediatric HO. The curative effect of antibiotic-impregnated Calcium Sulfate in pediatric ostemyelitis is satisfactory. We therefore recommend treating these patients with antibiotic-impregnated CS.

\section{Abbreviations}

HO, Hematogenous Osteomyelitis; CS, Calcium Sulfate; WBC, White Blood Cells; ESR, Erythrocyte Sedimentation Rate(ESR); CRP, C-Reactive Protein; MRI, Magnetic resonance imaging; Polymethylmethacrylate, PMMA.

\section{Declarations}

\section{Ethics approval and consent to participate}

Medical Ethics Committee of Nanfang Hospital of Southern Medical University has approved the research. The patients' parents agreed to participate in this study and a signed consent form was obtained from the patient's parents prior to the study. All procedures were conducted according to the Declaration of Helsinki.

\section{Consent for publication}

Written informed consent has been obtained from the parent for publication of this case report and any accompanying images. A copy of the written consent is available for review by the Editor of this journal.

\section{Availability of data and materials}

The data supporting our findings comes from Southern Medical University Nanfang hospital. The images and data sets used in the current study are available from the corresponding author if necessary.

\section{Competing interests}

The authors declare that they have no competing interests.

\section{Funding}

This study was not externally founded.

\section{Authors' contributions}

$\mathrm{CQ}, \mathrm{RT}$ and $\mathrm{CY}$ contributed equally to this work. Scientific idea: CQ, RT, CY; Project planning: CQ, RT, CY, JL, LX, JF, YH, CZ. Date collecton: RT, JL, LX, JF, CZ; Manuscript writing: CQ, RT, JL,YH; All authors read and approved the final manuscript. 
We would like to thank all the people who helped us in the current study.

\section{References}

1. Ma YF, Jiang N, Zhang $X$, Qin $C H$, Wang L, Hu YJ, Lin QR, Yu B, Wang BW: Calcium sulfate induced versus PMMA-induced membrane in a critical-sized femoral defect in a rat model. Sci Rep 2018, 8(1):637.

2. Jiang N, Qin CH, Ma YF, Wang L, Yu B: Possibility of one-stage surgery to reconstruct bone defects using the modified Masquelet technique with degradable calcium sulfate as a cement spacer: A case report and hypothesis. Biomed Rep 2016, 4(3):374-378.

3. Alexander DI, Manson NA, Mitchell MJ: Efficacy of calcium sulfate plus decompression bone in lumbar and lumbosacral spinal fusion: preliminary results in $\mathbf{4 0}$ patients. CAN J SURG 2001, 44(4):262-266.

4. Gitelis S, Piasecki P, Turner T, Haggard W, Charters J, Urban R: Use of a calcium sulfate-based bone graft substitute for benign bone lesions. ORTHOPEDICS 2001, 24(2):162-166.

5. Kelly CM, Wilkins RM, Gitelis S, Hartjen C, Watson JT, Kim PT: The use of a surgical grade calcium sulfate as a bone graft substitute: results of a multicenter trial. Clin Orthop Relat Res 2001(382):4250 .

6. Mirzayan R, Panossian V, Avedian R, Forrester DM, Menendez LR: The use of calcium sulfate in the treatment of benign bone lesions. A preliminary report. J BONE JOINT SURG AM 2001, 83(3):355358.

7. McKee MD, Li-Bland EA, Wild LM, Schemitsch EH: A prospective, randomized clinical trial comparing an antibiotic-impregnated bioabsorbable bone substitute with standard antibiotic-impregnated cement beads in the treatment of chronic osteomyelitis and infected nonunion. J ORTHOP TRAUMA 2010, 24(8):483-490.

8. Luo S, Jiang T, Yang Y, Yang X, Zhao J: Combination therapy with vancomycin-loaded calcium sulfate and vancomycin-loaded PMMA in the treatment of chronic osteomyelitis. BMC Musculoskelet Disord 2016, 17(1):502.

9. Jiang N, Ma YF, Jiang Y, Zhao XQ, Xie GP, Hu YJ, Qin CH, Yu B: Clinical Characteristics and Treatment of Extremity Chronic Osteomyelitis in Southern China: A Retrospective Analysis of 394 Consecutive Patients. Medicine (Baltimore) 2015, 94(42):e1874.

10. Andreacchio A, Alberghina F, Paonessa M, Cravino M, De Rosa V, Canavese F: Tobramycinimpregnated calcium sulfate pellets for the treatment of chronic osteomyelitis in children and adolescents. J PEDIATR ORTHOP B 2019, 28(3):189-195.

11. Lew DP, Waldvogel FA: Osteomyelitis. N Engl J Med 1997, 336(14):999-1007.

12. Funk SS, Copley LA: Acute Hematogenous Osteomyelitis in Children: Pathogenesis, Diagnosis, and Treatment. Orthop Clin North Am 2017, 48(2):199-208. 
13. Whyte NS, Bielski RJ: Acute Hematogenous Osteomyelitis in Children. PEDIATR ANN2016, 45(6):e204-e208.

14. Bachur R, Pagon Z: Success of short-course parenteral antibiotic therapy for acute osteomyelitis of childhood. Clin Pediatr (Phila) 2007, 46(1):30-35.

15. Lazzarini L, Lipsky BA, Mader JT: Antibiotic treatment of osteomyelitis: what have we learned from 30 years of clinical trials? INT J INFECT DIS 2005, 9(3):127-138.

16. Simpson AH, Deakin M, Latham JM: Chronic osteomyelitis. The effect of the extent of surgical resection on infection-free survival. J Bone Joint Surg Br 2001, 83(3):403-407.

17. Qin $\mathrm{CH}$, Zhou CH, Ren Y, Cheng GY, Zhang HA, Fang J, Tao R: Extensive eggshell-like debridement technique plus antibiotic-loaded calcium sulphate for one-stage treatment of chronic calcaneal osteomyelitis. FOOT ANKLE SURG 2020, 26(6):644-649.

18. DeRonde KJ, Girotto JE, Nicolau DP: Management of Pediatric Acute Hematogenous Osteomyelitis, Part l: Antimicrobial Stewardship Approach and Review of Therapies for Methicillin-Susceptible Staphylococcus aureus, Streptococcus pyogenes, and Kingella kingae. PHARMACOTHERAPY 2018, 38(9):947-966.

19. Athey AG, Mignemi ME, Gheen WT, Lindsay EA, Jo CH, Copley LA: Validation and Modification of a Severity of Illness Score for Children With Acute Hematogenous Osteomyelitis. J Pediatr Orthop 2019, 39(2):90-97.

20. Copley LA: Pediatric musculoskeletal infection: trends and antibiotic recommendations. J Am Acad Orthop Surg 2009, 17(10):618-626.

21. Jaeblon T: Polymethylmethacrylate: properties and contemporary uses in orthopaedics. J Am Acad Orthop Surg 2010, 18(5):297-305.

22. Zhang Z, Zhang C, Guo QF, Shen LF, Zhang XW: [Application of vancomycin-loaded calcium sulphate in treatment of osteomyelitis]. Zhongguo Yi Xue Ke Xue Yuan Xue Bao 2013, 35(3):337-342.

23. Wahl P, Guidi M, Benninger E, Rönn K, Gautier E, Buclin T, Magnin JL, Livio F: The levels of vancomycin in the blood and the wound after the local treatment of bone and soft-tissue infection with antibiotic-loaded calcium sulphate as carrier material. BONE JOINT J 2017, 99-B(11):15371544.

24. Davis WT, Gilbert SR: Comparison of Methicillin-resistant Versus Susceptible Staphylococcus aureus Pediatric Osteomyelitis. J Pediatr Orthop 2018, 38(5):e285-e291.

25. Anagnostakos $\mathrm{K}$, Wilmes $\mathrm{P}$, Schmitt $\mathrm{E}$, Kelm J: Elution of gentamicin and vancomycin from polymethylmethacrylate beads and hip spacers in vivo. ACTA ORTHOP 2009, 80(2):193-197.

26. Barrett RJ, Sandquist L, Richards BF, Soo TM: Antibiotic-impregnated polymethylmethacrylate as an anterior biomechanical device for the treatment of cervical discitis and vertebral osteomyelitis: technical report of two cases. TURK NEUROSURG 2014, 24(4):613-617.

27. Güler UO, Derincek A, Hersekli MA, Ozalay M, Cinar BM, Acaroğlu E: Restoration of pull-out strength of the failed pedicle screw: biomechanical comparison of calcium sulfate vs polymethylmethacrylate augmentation. Acta Orthop Traumatol Turc 2014, 48(2):202-206. 
28. Helgeson MD, Potter BK, Tucker CJ, Frisch HM, Shawen SB: Antibiotic-impregnated calcium sulfate use in combat-related open fractures. ORTHOPEDICS 2009, 32(5):323.

29. Rauschmann MA, Wichelhaus TA, Stirnal V, Dingeldein E, Zichner L, Schnettler R, Alt V: Nanocrystalline hydroxyapatite and calcium sulphate as biodegradable composite carrier material for local delivery of antibiotics in bone infections. BIOMATERIALS 2005, 26(15):2677-2684.

30. McKee MD, Wild LM, Schemitsch EH, Waddell JP: The use of an antibiotic-impregnated, osteoconductive, bioabsorbable bone substitute in the treatment of infected long bone defects: early results of a prospective trial. J ORTHOP TRAUMA 2002, 16(9):622-627.

31. Ferguson JY, Dudareva M, Riley ND, Stubbs D, Atkins BL, McNally MA: The use of a biodegradable antibiotic-loaded calcium sulphate carrier containing tobramycin for the treatment of chronic osteomyelitis: a series of 195 cases. BONE JOINT J 2014, 96-B(6):829-836.

32. Azi ML, Teixeira A, Cotias RB, Joeris A, Kfuri M: Induced-Membrane Technique in the Management of Posttraumatic Bone Defects. JBJS Essent Surg Tech 2019, 9(2):e22.

33. Canavese F, Corradin M, Khan A, Mansour M, Rousset M, Samba A: Successful treatment of chronic osteomyelitis in children with debridement, antibiotic-laden cement spacer and bone graft substitute. Eur J Orthop Surg Traumato/2017, 27(2):221-228.

34. Bar-On E, Weigl DM, Bor N, Becker T, Katz K, Mercado E, Livni G: Chronic osteomyelitis in children: treatment by intramedullary reaming and antibiotic-impregnated cement rods. J Pediatr Orthop 2010, 30(5):508-513.

\section{Figures}






Figure 1

Diagram of included patients. 


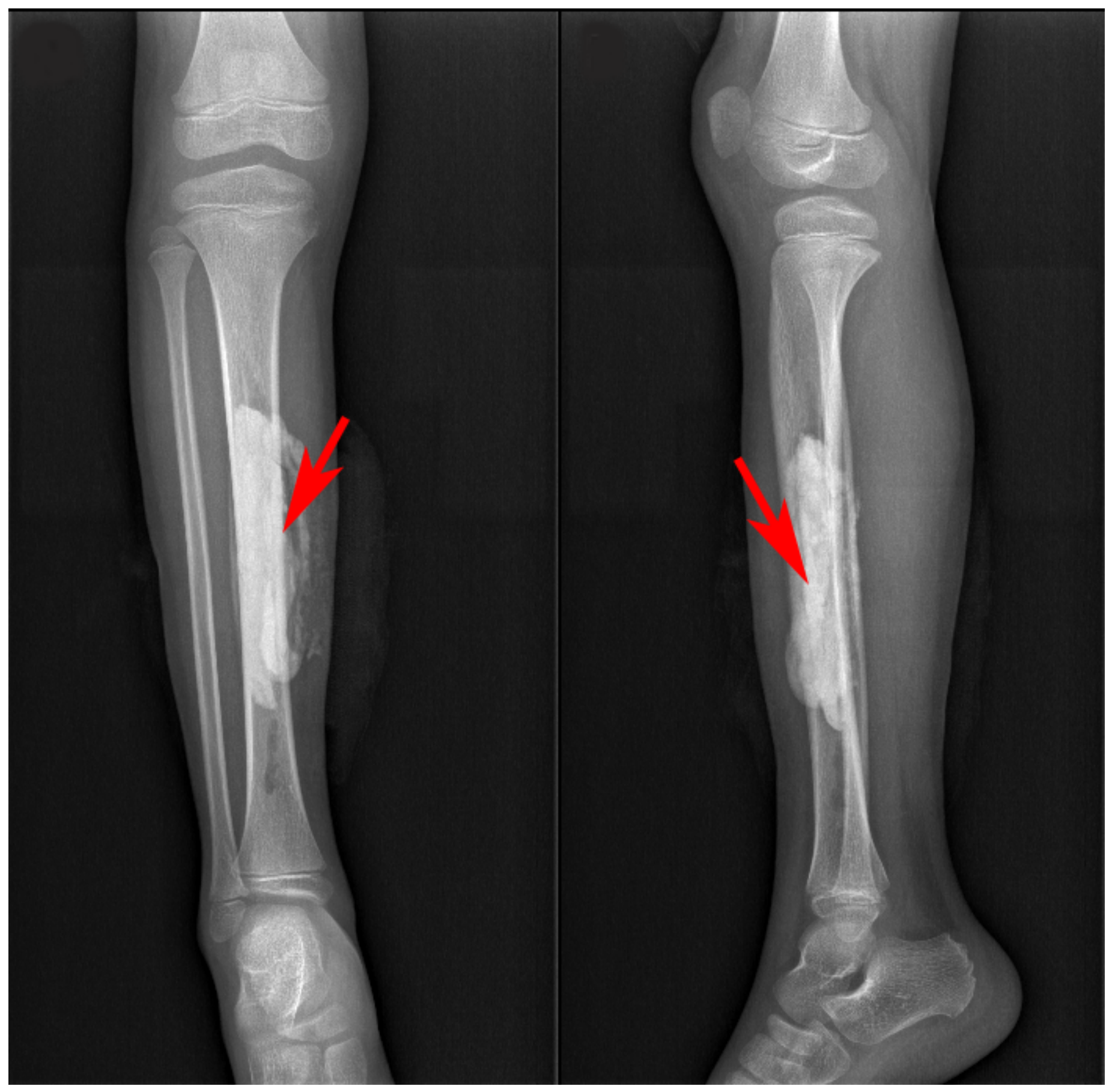

Figure 2

Antibiotic-impregnated calcium sulfate was applied to obliterate the dead space and release local antibiotic in tibial hematogenous osteomyelitis. The red arrows indicate calcium sulfate. 


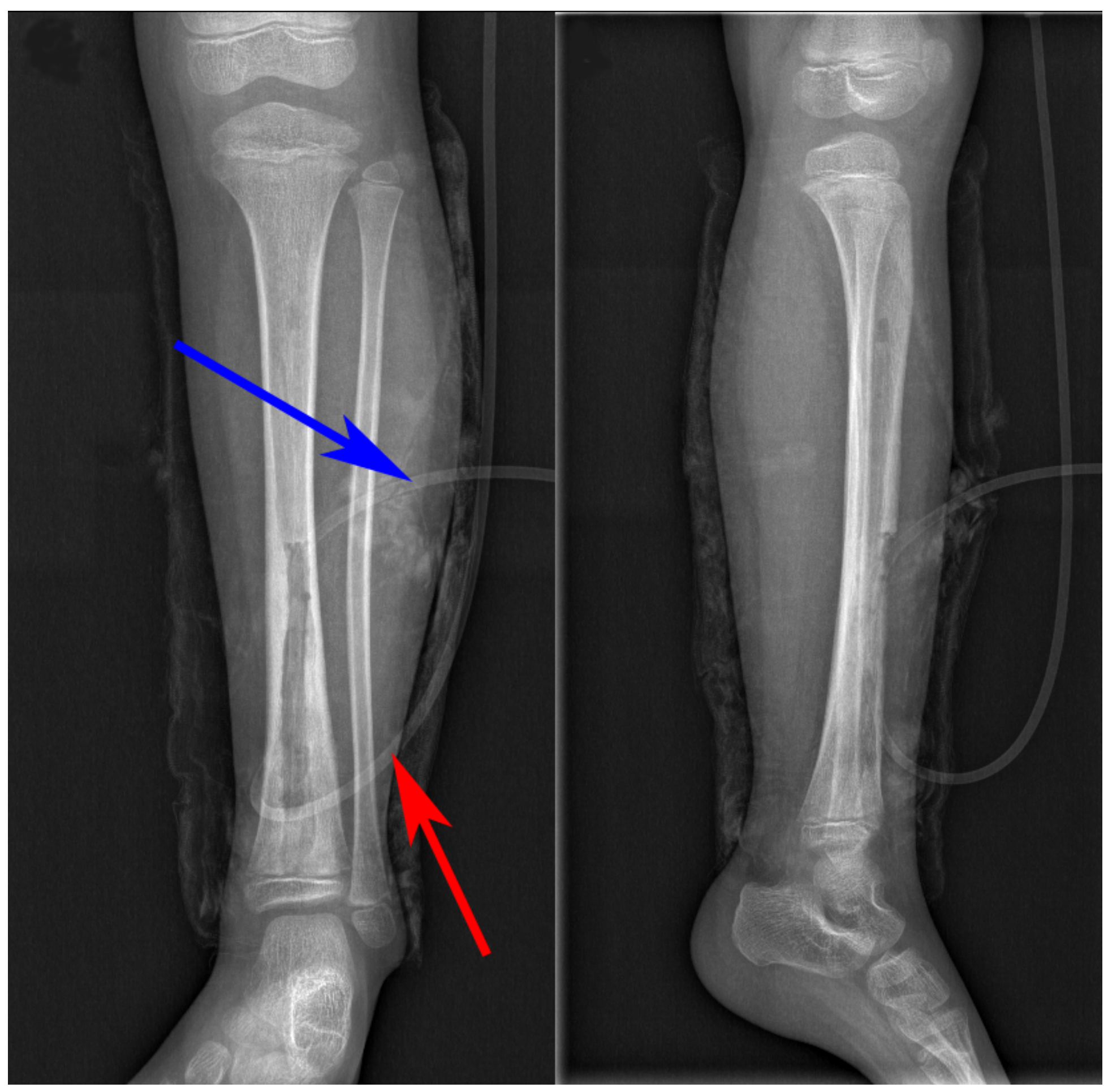

\section{Figure 3}

Decompression surgery alone in tibia hematogenous osteomyelitis. A irrigation tube was placed at proximal tibia and a drain tube was placed at distal tibia. The red arrow indicate irrigation tube, the blue arrow indicate drain tube. 

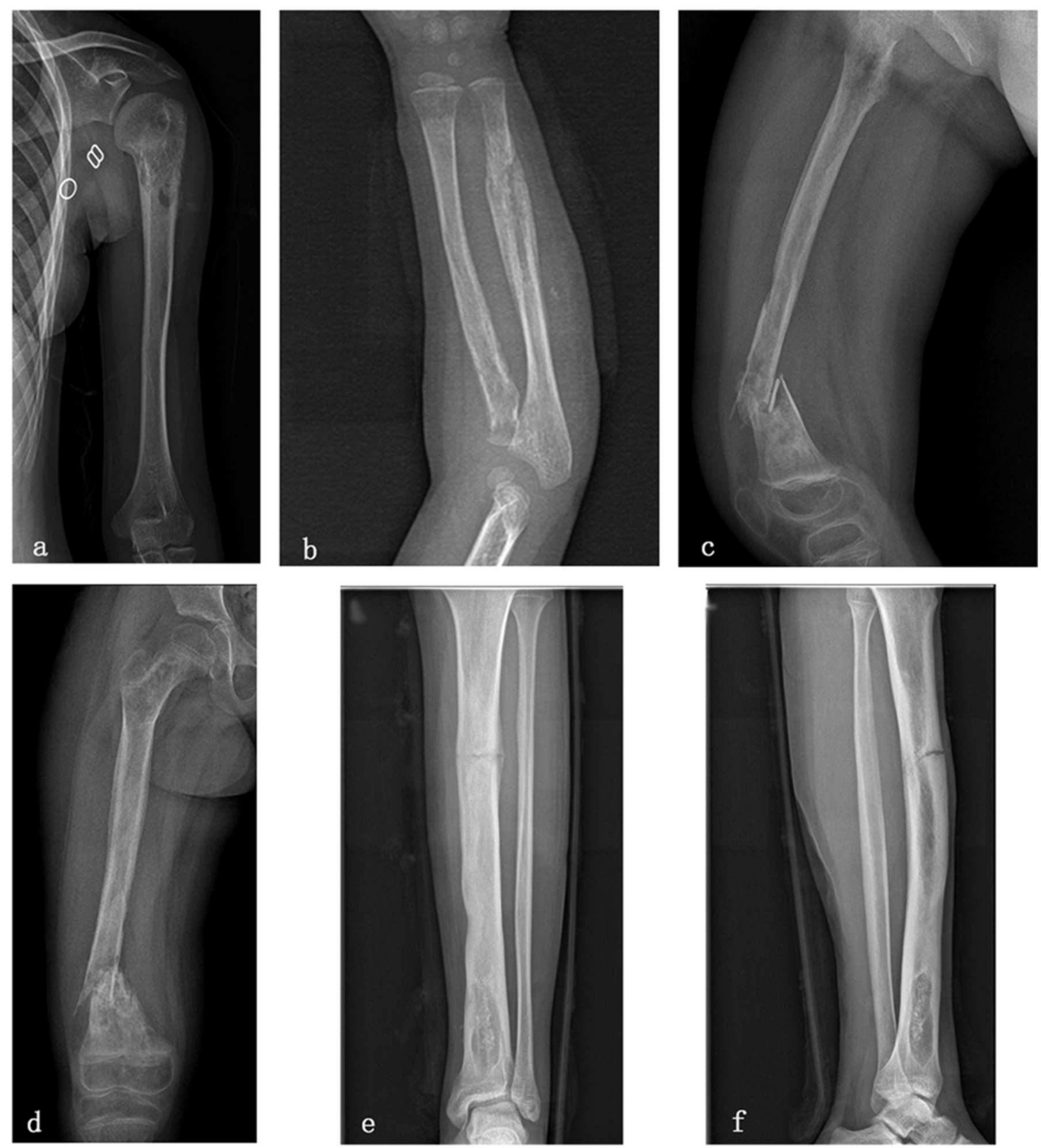

\section{Figure 4}

a 5 years old girl who underwent a decompression surgery in her left humerus and explosive effusion from the wound postoperation; $b$ a 2 years old girl who underwent a decompression surgery in her left ulnar and explosive effusion from the wound postoperation; c-d a 5 years old girl who underwent a decompression surgery in her right femur and pathological fracture postoperation; e-f a 14 years old boy who underwent a decompression surgery in his left tibial and pathological fracture postoperation. 